\title{
Combinatorial identities in number theory related to $q$-series and arithmetical functions
}

\section{Geoffrey Bruce Campbell}

This thesis introduces a variety of new results in number theory based upon two related themes. Specifically, the first theme relates to a theory of partitions, and especially to visible point vector identities as given in the author's papers. The second theme involves a $D$-analogue or Dirichlet series analogue for basic hypergeometric series. Both themes are related by dependence on hypergeometric series.

The first theme includes some functional equations, and later embraces a new class of combinatorial identities with many applications to classical partition theory. It involves also some studies of arithmetical functions.

A typical identity is: If $|x y z|,|y z|$ and $|z|<1$, and $r+s+t=1$, then

$$
\begin{aligned}
& \exp \left\{\sum_{k \geqslant 1} \frac{z^{k}}{k^{t}}\right\} \prod_{\substack{(a, b, c)=1 \\
a, b<c \\
a, b>0 ; c>1}}\left(1-x^{a} y^{b} z^{c}\right)^{-a^{-r} b^{-s} c^{-t}} \\
& \quad=\exp \left\{\frac{z^{1}}{1^{t}}+\left(1+\frac{x^{1}}{1^{r}}\right)\left(1+\frac{y^{1}}{1^{s}}\right) \frac{z^{2}}{2^{t}}\left(1+\frac{x^{1}}{1^{r}}+\frac{x^{2}}{2^{r}}\right)\left(1+\frac{y^{1}}{1^{s}}+\frac{y^{2}}{2^{s}}\right) \frac{z^{3}}{3^{t}}+\ldots\right\}
\end{aligned}
$$

Hence chapters two to four relate more to theory of partitions. We continue previous researches on partition identities with extensions and added concepts which will of themselves lead to new researches. For example, new generalizations of the classical Ramanujan arithmetical function are given, and results related to vector or multipartite number partitions.

The second theme involves a new Dirichlet series analogue for the already known and well researched hypergeometric $q$-series, often called the basic hypergeometric series. The basic hypergeometric series, whilst widely used in mathematics, is itself an analogue for the ordinary hypergeometric series developed by Gauss in the early nineteenth century.

Received 9th April, 1998

Thesis submitted to Australian National University, October 1997. Degree approved, March 1998. Supervisor: Professor Rodney J. Baxter.

Copyright Clearance Centre, Inc. Serial-fee code: 0004-9729/98 \$A2.00+0.00. 
A comparison shows that the original Gauss series is

$$
1+\frac{a b}{c 1 !} z+\frac{a(a+1) b(b+1)}{c(c+1) 2 !} z^{2}+\frac{a(a+1)(a+2) b(b+1)(b+2)}{c(c+1)(c+2) 3 !} z^{3}+\ldots
$$

whilst the $q$-series due to Heine last century is

$$
\begin{aligned}
1+\frac{(1-a)(1-b)}{(1-c)(1-q)} z+ & \frac{(1-a)(1-a q)(1-b)(1-b q)}{(1-c)(1-c q)(1-q)\left(1-q^{2}\right)} z^{2} \\
& +\frac{(1-a)(1-a q)\left(1-a q^{2}\right)(1-b)(1-b q)\left(1-b q^{2}\right)}{(1-c)(1-c q)\left(1-c q^{2}\right)(1-q)\left(1-q^{2}\right)\left(1-q^{3}\right)} z^{3}+\ldots
\end{aligned}
$$

and the $D$-analogue given in the author's papers just predating this thesis is

$$
\sum_{k=1}^{\infty}\left(\prod_{j=0}^{a-2} \frac{\sigma_{-\gamma}\left(k \prod_{p \mid k} p^{j}\right)}{\sigma_{-\gamma}\left(\prod_{p \mid k} p^{j}\right)}\right)\left(\prod_{j=0}^{b-2} \frac{\sigma_{-\gamma}\left(k \prod_{p \mid k} p^{j}\right)}{\sigma_{-\gamma}\left(\prod_{p \mid k} p^{j}\right)}\right)\left(\prod_{j=0}^{c-2} \frac{\sigma_{-\gamma}\left(\prod_{p \mid k} p^{j}\right)}{\sigma_{-\gamma}\left(k \prod_{p \mid k} p^{j}\right)}\right) \frac{1}{k^{z}}
$$

in which we incorporate the sum of powers of divisors function

$$
\sigma_{-\gamma}(k)=\frac{\sigma_{\gamma}(k)}{k^{\gamma}}
$$

This notation allows generalization to a multidimensional divisor function in the Dirichlet series coefficients.

We therefore, onward from Chapter five, examine a new class of Dirichlet series based on the already known and well established $q$-series, and on a new class of similar Dirichlet series already discovered by the author. An example of the recent research slightly predating this thesis work is the series for positive integers $n$

$$
\sum_{k=1}^{\infty}\left(\prod_{j=0}^{n-1} \frac{\sigma_{-\gamma}\left(k \prod_{p \mid k} p^{j}\right)}{\sigma_{-\gamma}\left(\prod_{p \mid k} p^{j}\right)}\right) \frac{1}{k^{\beta}}=\prod_{k=0}^{n} \zeta(\beta+k \gamma), \quad \Re \beta>1, \Re \gamma>0 .
$$

Throughout this thesis the Riemann zeta function and the sum of powers of the divisors function appear. Similar summations are given for analogues of the $q$-Gauss, $q$-Kummer, and the $q$-Dixon sums.

In the author's papers $[1,2]$ these $D$-analogues were found at first from applying an Euler product operator over all primes, then later analogues came from application of a partial product in the operator. This later rationale leads to a distinct class of $D$-analogues. The key to this is as follows. Assume the positive integer prime 
decomposition $m=\prod_{i=1}^{s} p_{i}^{a_{i}}$, and that we associate with $m$ a set $S_{m}=\left\{x \in Z^{+}\right.$: $x=\prod_{i=1}^{s} p_{i}^{b_{i}}$ where $\left.b_{i} \in Z^{+}\right\}$. Then for positive integers $n$ we have, for example, a new $q$-binomial theorem analogue

$$
\sum_{k \in S_{m}}\left(\prod_{j=0}^{n-1} \frac{\sigma_{-\gamma}\left(k \prod_{p \mid k} p^{j}\right)}{\sigma_{-\gamma}\left(\prod_{p \mid k} p^{j}\right)}\right) \frac{\lambda(k)}{k^{\beta}}=\prod_{k=0}^{n} \frac{1}{\sigma_{-(\beta+k \gamma)}\left(\prod_{p \mid m} p\right)}, \quad \mathfrak{R} \beta>1, \mathfrak{R} \gamma>0,
$$

where $\lambda$ is the Liouville function

$$
\lambda(k)=\prod_{i=1}^{t}(-1)^{a_{i}} \text { for each } k=\prod_{i=1}^{t} p_{i}^{a_{i}} .
$$

Again, summations are given as analogues for the $q$-Gauss, $q$-Kummer, and the $q$-Dixon sums.

A natural extension of the ideas presented so far is in the defining of $D$-analogues for the $q$-gamma and $q$-beta functions. Chapter 9 describes the likely forms of the $D$-series analogues and gives corresponding results for the clssical $q$-gamma and $q$-beta functions.

\section{REFERENCES}

[1] G.B. Campbell, 'Dirichlet series analogues of $q$-series', Internat J. Math. Math. Sci. (to appear).

[2] G.B. Campbell, 'Further Dirichlet series analogues of $q$ - series', Internat J. Math. Math. Sci. (to appear).

School of Mathematical Sciences

Australian National University

Canberra ACT 0200

Australia 